\title{
Protagonismo de la administración de empresas en la implementación de las normas de calidad ISO 9001: 2015
}

\section{Protagonism of business administration in the implementation of quality standards ISO 9001: 2015}

\section{Protagonismo da administração de empresas na implementação de padrões de qualidade ISO 9001: 2015}

\author{
Harold S. Quiñonez-Francis ${ }^{\mathrm{I}}$ \\ hasqui@hotmail.com \\ Marcelo E. Reina-Tello II \\ marceloreinatello1@ hotmail.com \\ Félix A. Bravo-Mendoza ${ }^{\text {III }}$ \\ josephbravojama@gmail.com
}

Recibido: 18 de mayo de 2017 * Corregido: 30 de julio de 2017 * Aceptado: 12 de septiembre 2017

I Magister en Educación Mención en Psicodidáctica, Especialista en Derecho Procesal Penal, Magister en Administración de Empresas, Diplomado Superior en Docencia Universitaria, Diplomado Superior en Gestión Integral de Riesgos y Desastres, Abogado de los Tribunales y Juzgados de la República del Ecuador, Docente de la Universidad Técnica Luis Vargas Torres de Esmeraldas, Esmeraldas, Ecuador.

II Licenciado en Administración de Empresas, Ingeniero Comercial, Magister en Administración de Empresas, Universidad Técnica Luis Vargas Torres de Esmeraldas, Esmeraldas, Ecuador.

III Ingeniero Comercial, Msc. Docencia Universitaria Mención Desarrollo del Currículo, Docente de la Universidad Técnica Luis Vargas Torres de Esmeraldas, Esmeraldas, Ecuador. 


\section{Resumen}

Un Sistema de Gestión de la Calidad requiere el apoyo de la dirección. Específicamente la ISO 9001:2015 implica totalmente a la alta dirección. Este trabajo tiene como objetivo mostrar el protagonismo de la administración de empresas en la implementación de las Normas de Calidad ISO 9001 del 2015. Para ello se hace un análisis del contenido de la Norma ISO 9001: 2015 y se revisa la literatura publicada sobre el tema.

Palabras clave: administración de empresas; ISO 9001:2015; normas de calidad; sistema de gestión de la calidad; alta dirección.

\section{Abstract}

A Quality Management System requires the support of the management. Specifically, ISO 9001: 2015 fully involves top management. This work aims to show the role of business administration in the implementation of ISO 9001 Quality Standards of 2015. For this purpose, an analysis of the content of ISO 9001: 2015 is made and the literature published on the subject is reviewed.

Keywords: business administration; ISO 9001: 2015; quality standards; quality management system; high direction.

\section{Resumo}

Um Sistema de Gestão da Qualidade requer o suporte da gestão. Especificamente, o ISO 9001: 2015 envolve completamente a alta administração. Este trabalho tem como objetivo mostrar o papel da administração de empresas na implementação dos Padrões de Qualidade ISO 9001 de 2015. Para isso, é feita uma análise do conteúdo da ISO 9001: 2015 e a literatura publicada sobre o assunto é revisada.

Palavras chave: administração de empresas; ISO 9001: 2015; padrões de qualidade; sistema de gestão da qualidade; alta direção.

\footnotetext{
544 Vol. 3, núm 4, octubre, 2017, pp. 543-554

Harold S. Quiñonez Francis, Marcelo E. Reina Tello, Félix A. Bravo Mendoza
} 


\section{Introducción}

A lo largo de la vida de ISO 9001 y de sus revisiones, el papel de los Representantes de Dirección, requisito obligatorio, ha ido cambiando. En cada revisión, este papel ha ido sufriendo unas modificaciones: (Escuela Europea de Excelencia, 2013)

- ISO 9001:2000. Era la organización quien debía designar al Representante de la Dirección.

- ISO 9001:2008. En esta revisión se añade un nuevo requisito, el Representante de la Dirección debe ser un miembro de la dirección de la organización.

- ISO 9001:2015. El Comité de proyectos asigna el rol de Representante de la Dirección a la alta dirección de la organización y no requiere que una persona específica sea asignada con este papel.

En la nueva versión ISO 9001:2015 la alta dirección cumplirá con las responsabilidades que antes eran asignadas al Representante de la Dirección.

Con esta nueva versión desaparece la figura del Representante de la Dirección. Esto se debe a la intención de que las responsabilidades de la alta dirección con el sistema no se diluyan, sino que queden claras y definidas. La norma en este caso aporta flexibilidad, pues permite que la empresa siga delegando y manteniendo la figura del Representante de la Dirección con las principales responsabilidades de la anterior norma ISO 9001:2008, aunque ya sin ser un requisito. (Gehisy, 2016)

Un SGC sin el apoyo de la dirección está condenado al fracaso. La ISO 9001:2015 implica totalmente a la alta dirección. La administración de las empresas juega un papel protagónico en la implementación de las Normas de Calidad ISO 9001 del 2015. (ISO, 2015)

Este trabajo tiene como objetivo mostrar el protagonismo de la administración de empresas en la implementación de las Normas de Calidad ISO 9001 del 2015.

Para ello se hace un análisis del contenido de la Norma ISO 9001: 2015 y se revisa la literatura publicada sobre el tema.

\footnotetext{
545 Vol. 3, núm 4, octubre, 2017, pp. 543-554

Harold S. Quiñonez Francis, Marcelo E. Reina Tello, Félix A. Bravo Mendoza
} 


\section{Desarrollo}

Según la norma, la alta dirección es la persona o grupo de personas que dirige y controla una organización al más alto nivel. No obstante, puede delegar funciones y proporcionar los recursos necesarios para alcanzar los objetivos de calidad, sin olvidar que el éxito o el fracaso de sus decisiones recaen bajo su responsabilidad. (CAVALA, 2015)

La alta dirección tiene claramente asignadas responsabilidades, pero como siempre, que sea responsable de que el trabajo se haga no quiere decir que lo tenga que hacer ella directamente. Lo que sí es cierto es que, el enfoque a procesos de la revisión 2015 de la norma es mucho más 'profundo' y al final va a obligar a que existan responsables de procesos, 'dueños de los procesos' como se les suele llamar, y como se ha de integrar la calidad en los procesos, los Responsables de Calidad, por fin, van a poder realizar una labor de gestión, análisis y recomendaciones para la mejora. (HAZA, 2016)

El principal cambio en la nueva norma ISO 90012015 con respecto a la norma anterior, reside en el hecho de que la dirección no sólo debe asegurarse de proveer los recursos para que el sistema de calidad funcione, sino que además debe demostrar su implicación en las actividades del sistema de gestión en toda su amplitud. Los mecanismos a través de los cuales la alta dirección puede alcanzar estos objetivos son: (CAVALA, 2015)

- El compromiso y liderazgo de la alta dirección con el sistema de calidad.

- El compromiso y liderazgo con el cliente.

- El establecimiento, la revisión y el mantenimiento de una política de calidad.

- La asignación de las responsabilidades y autoridades para los roles pertinentes, así como que sean comunicados y entendidos dentro de la organización.

Uno de los problemas que han tenido las normas ISO, era falta de implicación de la dirección de la organización en el sistema de gestión. Parece que la intención de la nueva versión de la norma es

\footnotetext{
546 Vol. 3, núm 4, octubre, 2017, pp. 543-554

Harold S. Quiñonez Francis, Marcelo E. Reina Tello, Félix A. Bravo Mendoza
} 
acabar con este problema, ya que ahora la dirección será la responsable de integrar los requisitos del sistema de gestión en los procesos de la organización, asegurar que el sistema logra los resultados deseados y asignar los recursos que hagan falta. Ahora, la alta dirección también es responsable de comunicar la importancia del sistema de gestión y aumentar la toma de conciencia y la participación de los empleados. El liderazgo en la norma ISO 9001:2015 se entiende como la necesidad de que la alta dirección tome la iniciativa, gestione, promueva y evalúe de forma eficaz y eficiente el logro de los resultados deseados. (HAZA, 2016)

Tras la revisión el pasado 2015 de la norma ISO 9001, son varios los cambios y novedades introducidos. Tal es el caso del liderazgo en ISO 9001:2015. Respecto a ello, la nueva versión de la norma enfatiza la mayor involucración necesaria por parte de la Alta Dirección en lo que a Gestión de la Calidad se refiere. Concretamente, es en la cláusula 5, se recoge todas las novedades introducidas en referencia al liderazgo en ISO 9001:2015. Entre tales aspectos que la nueva norma considera destacamos: (HUBSPot, 2016)

- La importancia de contar con políticas, funciones de responsabilidad, autoridades y responsabilidades en la organización.

- Realza la responsabilidad que tiene la Alta Dirección dentro del Sistema de Gestión de Calidad (SGC), debiendo demostrar su fuerte compromiso con el mismo.

Cuando hablamos de liderazgo en ISO 9001:2015 nos referimos al conjunto de capacidades que deben estar presentes en una persona para lograr ejercer influencia sobre un grupo de personas, con el fin de motivarlas y conducirlas hacia el logro de unos determinados objetivos propuestos. (HUBSPot, 2016)

La alta dirección tiene que estar motivada, decidida y plantearse la implantación del SGC como un objetivo estratégico. La alta dirección debe mostrar liderazgo y compromiso. El líder (o el jefe) de la organización no solo debe demostrar que lo es, sino que debe estar comprometido. ¿De qué manera?: (Gehisy, 2016)

547 Vol. 3, núm 4, octubre, 2017, pp. 543-554
Harold S. Quiñonez Francis, Marcelo E.

Harold S. Quiñonez Francis, Marcelo E. Reina Tello, Félix A. Bravo Mendoza 
- Alineando la calidad con los objetivos estratégicos de la organización.

- Siendo el motor del Sistema de Gestión (y no un estorbo, como desgraciadamente pasa a veces).

- Involucrando a todo el personal en el diseño, implantación y mejora del Sistema.

- Asegurando la disponibilidad de los recursos necesarios para cada proceso.

El liderazgo en la norma ISO 9001:2015 tiene un enfoque activo, frente al papel más pasivo que podría interpretarse en la revisión 2008. La implicación de la dirección no es opcional, ya que la revisión 2015 asigna la responsabilidad a la alta dirección de la organización de los objetivos de calidad estratégica, el alcance del SGC y los resultados, políticas y procesos, la comunicación, la cultura, el fomento de un compromiso con la calidad, de proporcionar recursos y oportunidades de formación, e incluso de inspirar, fomentar y reconocer la contribución de todos los miembros de la organización. (HAZA, 2016)

A partir de la literatura consultada, a continuación se expondrán las cuestiones que le incumben a la ALTA DIRECCIÓN, en relación a la ISO 9001 del 2015. La alta dirección debe:

- Decidir y promover la adopción de la ISO 9001 en la Organización.

- Demostrar su implicación en las actividades del sistema de gestión en toda su amplitud.

- Responsabilizarse con la eficacia del sistema de calidad.

- Marcar el rumbo y conducir a la Organización.

- Aclarar la razón de la existencia de la Organización.

- Asegurar que los resultados deseados del SGC son alcanzados a través del cumplimiento de sus funciones y de la aplicación de los requisitos de la norma de referencia a la Organización.

- Conferir unidad de propósito y establecer la dirección estratégica de la Organización.

\footnotetext{
548 Vol. 3, núm 4, octubre, 2017, pp. 543-554

Harold S. Quiñonez Francis, Marcelo E. Reina Tello, Félix A. Bravo Mendoza
} 
El término dirección estratégica aparece unas cinco veces en la nueva ISO 9001:2015, la versión antigua no lo menciona en ningún aspecto estratégico. (Toro R., 2015)

- Integrar en los procesos de negocio de la Organización al SGC.

Debe apoyar todas las funciones de gestión relevantes a la hora de demostrar liderazgo y compromiso, en sus respectivas áreas de actuación.

- Establecer una política de calidad y unos objetivos compatibles con la dirección estratégica y el contexto de la organización.

La ISO 9001 requiere que se asegure la alineación de la política y de los objetivos de la calidad con la intención estratégica o con cualquier formulación de estrategia eventualmente adoptada por la Organización. La estrategia puede ser visualizada como un conjunto de compromisos y acciones diseñadas para explorar las competencias clave de la Organización y ganar unas ventajas competitivas.

Se debe lograr que la política esté estrechamente relacionada con la Visión, Misión, Valores y Objetivos estratégicos de la empresa.

Es importante asociarla con los Objetivos de Calidad. La Política es la base y los Objetivos de Calidad serán la parte medible, por lo que deben ir de la mano.

- Asegurarse que la política de calidad se establezca, se revise, se mantenga, se comunique, sea entendida y se aplique.

Independientemente de los medios de comunicación usados, es importante asegurar que la Organización percibe ese mensaje como proveniente de la alta dirección.

La política según la Norma ISO 9001:2015 debe ser adecuada al propósito y contexto de la organización, que proporcione un marco para establecer y revisar los objetivos de la calidad, que incluya un compromiso de cumplir con los requisitos aplicables y un compromiso de mejora continua del sistema de gestión de calidad.

\section{Vol. 3, núm 4, octubre, 2017, pp. 543-554}

Harold S. Quiñonez Francis, Marcelo E. Reina Tello, Félix A. Bravo Mendoza 
Esto quiere decir que no se debe copiar y pegar de otras Políticas de Calidad pues cada organización es diferentes.

Se incluye de manera novedosa en esta versión de la norma 2015 que la Política de Calidad debe entenderse y aplicarse, así como estar disponible para las partes interesadas.

- Debe asignar la responsabilidad y autoridad para:

a) Garantizar que el sistema de gestión de calidad es conforme con los requisitos de la Norma Internacional ISO 9001:2015.

b) Garantizar que los procesos consiguen los resultados previstos.

c) Informar sobre el desempeño del sistema de gestión de calidad, sobre las oportunidades de mejora y sobre las necesidades de cambio o innovación, y sobre todo para informar a la alta dirección.

d) Garantizar la promoción de la orientación al cliente en toda la organización.

e) Asegurar que la integridad del sistema de gestión de la calidad se mantiene cuando se planifican e implementan cambios en el sistema de gestión de calidad.

- Decidir el nivel de autonomía de las personas.

- Crear las condiciones para que las personas, en la Organización, se comprometan para alcanzar los objetivos de la calidad definidos.

El principio de calidad número 2 según la Norma ISO 9001 del 2015 plantea "Los líderes de las Organizaciones definen el propósito y la dirección de la Organización, y crean las condiciones para que la Organización, como un todo camine en esa dirección y trabaje para alcanzar los resultados deseados, condición sin la cual difícilmente se obtendrá éxito. Las personas hacen las cosas suceder. Para lograr los objetivos, es necesario que las personas actúen como un todo, orientadas por los mismos objetivos. Las personas deben ser lideradas en este camino y disponer de las condiciones recursos, competencia y conocimiento - para lograrlos. Es esencial que las personas de la 
Dom. Cien., ISSN: $2477-8818$

Vol. 3, núm. 4, octubre, 2017, pp. 543-554

Protagonismo de la administración de empresas en la implementación de las normas de calidad ISO 9001: 2015

Organización conozcan y comprendan su propósito y dirección, y se sientan apoyadas y estimuladas en su consecución".

- Promover el enfoque de procesos.

- Promover la adopción del ciclo PHVA para la mejora.

- Promover el enfoque basado en riesgos.

- Reforzar y comunicar la importancia de una gestión de la calidad eficaz y en conformidad con los requisitos.

- Proveer los recursos para que el sistema de calidad funcione y decidir sobre la mejor forma de utilizar esos recursos.

- Orientar y apoyar a las personas de la Organización para alcanzar los resultados deseados del SGC.

- Representar legalmente a la Organización, respondiendo por la misma.

- Representar a la Organización frente a terceros

- Asegurar que los productos y servicios que se ofrecen a los clientes cumplan con los aspectos legales aplicables y los requisitos reglamentarios.

- Asegurar que se determinan y se tratan los riesgos (amenazas) y oportunidades que pueden afectar a la conformidad de los productos y servicios, y se satisfaga al cliente.

Ya no vale solamente con intenciones, es requisito que se identifiquen los requisitos del cliente, los riesgos y oportunidades inherentes a estos y que no se pierda de vista que se debe aumentar la satisfacción del cliente.

- Asegurar que todos conocen esos resultados deseados y caminan en la misma dirección, con compromiso y responsabilidad.

- Asumir la responsabilidad por las consecuencias de los fallos en los productos y servicios.

\section{Vol. 3, núm 4, octubre, 2017, pp. 543-554}

Harold S. Quiñonez Francis, Marcelo E. Reina Tello, Félix A. Bravo Mendoza 
Dom. Cien., ISSN: $2477-8818$

Vol. 3, núm. 4, octubre, 2017, pp. 543-554

Protagonismo de la administración de empresas en la implementación de las normas de calidad ISO 9001: 2015

- Rendir cuentas del funcionamiento del SGC. Revisar la adecuación y eficacia del SGC.

Seguir el logro de los objetivos, el grado de cumplimiento de las disposiciones planificadas y su conformidad con los requisitos de la norma a través de las prácticas de revisión por la dirección.

La alta dirección debe revisar el sistema de gestión de calidad de la organización, a intervalos planificados, para asegurar su continua conveniencia, adecuación y eficacia.

La revisión por la dirección debe ser planificada y llevada a cabo teniendo en cuenta:

a) El estado de las acciones de las revisiones por la dirección previas.

b) Los cambios en las cuestiones externas e internas que son relevantes para el sistema de gestión de calidad y para su dirección estratégica.

c) Información sobre el funcionamiento de la calidad, incluyendo las tendencias e indicadores para:

- No conformidades y acciones correctivas.

- Resultados del seguimiento y la medición.

- Resultados de las auditorías.

- La satisfacción del cliente.

- Cuestiones relativas a los proveedores externos y otras partes interesadas pertinentes.

- La adecuación de los recursos necesarios para mantener un eficaz sistema de gestión de calidad.

- Desempeño de los procesos y la conformidad de los productos y servicios.

d) La eficacia de las medidas adoptadas para abordar los riesgos y las oportunidades.

e) Nuevas oportunidades para la mejora continua.

552 Vol. 3, núm 4, octubre, 2017, pp. 543-554

Harold S. Quiñonez Francis, Marcelo E. Reina Tello, Félix A. Bravo Mendoza 
Dom. Cien., ISSN: $2477-8818$

Vol. 3, núm. 4, octubre, 2017, pp. 543-554

Protagonismo de la administración de empresas en la implementación de las normas de calidad ISO 9001: 2015

Los resultados de la revisión por la dirección debe incluir las decisiones y acciones relacionadas con:

a) Las oportunidades para la mejora continua.

b) Cualquier necesidad de cambio en el sistema de gestión de calidad, incluyendo las necesidades de recursos.

La organización debe retener la información documentada como evidencia de los resultados de las revisiones por la dirección.

\section{Conclusiones}

Este trabajo evidencia que la administración de empresas no puede estar divorciado del Sistema de Gestión de la Calidad. La norma ISO 9001:2015 da mayor protagonismo a la alta dirección y a los dueños de procesos, exige mayor compromiso y liderazgo.

"Pretende hacer a la alta dirección y a los dueños de procesos los partícipes directos de sus procesos, auditando, informando, midiendo indicadores e identificando activamente riesgos y oportunidades. De esta manera, la responsabilidad no recae solamente sobre una persona (como solía pasar con el Representante de la Dirección), sino que se da responsabilidad y autoridad a otros actores dentro del Sistema de Gestión”. (Gehisy, 2016)

\section{Referencias bibliográficas}

CAVALA. (2015). Claves-iso-9001-2015-liderazgo. Obtenido de http://www.cavala.es

Escuela Europea de Excelencia. (2013). ISO 9001: 2015. El papel de los representantes de la dirección. Obtenido de http://www.nueva-iso-9001-2015.com 
Dom. Cien., ISSN: $2477-8818$

Vol. 3, núm. 4, octubre, 2017, pp. 543-554

Protagonismo de la administración de empresas en la implementación de las normas de calidad ISO 9001: 2015

Gehisy. (2016). La ISO 9001:2015 punto por punto: Capítulo 5. Obtenido de http://aprendiendocalidadyadr.com

HAZA. (2016). El liderazgo en la norma ISO 9001:2015. Cláusula 5. ¿Quién se ha llevado mi ISO? Obtenido de http://www.hazaconsejerostecnicos.com

HUBSPot. (2016). Liderazgo en ISO 9001:2015. Obtenido de http://www.123aprende.com

Toro R. (2015). ISO 9001:2015 y la dirección estratégica. Obtenido de http://www.nueva-iso-90012015.com 